\title{
Abordagem cirúrgica de fechamento de fístula buco-sinusal pela técnica do retalho vestibular: relato de caso
}

\author{
Surgical approach to buccal-sinus fistula closure by vestibular flap technique: case report
Abordaje quirúrgico del cierre de la fístula del seno bucal mediante técnica de colgajo vestibular: reporte de caso

Flayane Nuberly Farias Gomes dos Anjos ${ }^{1 *}$, Maria Carolina Gaia de Melo ${ }^{1}$, Ingrid Ferreira Leite ${ }^{1}$, Diana Pimentel de Melo ${ }^{1}$, Luciano de Almeida Lucas ${ }^{1}$, Lucas Fortes Cavalcanti de Macedo', Tayguara Cerqueira Cavalcanti¹.

\section{RESUMO}

Objetivo: Elucidar acerca da abordagem cirúrgica do fechamento de fístula buco-sinusal pela técnica de retalho vestibular, bem como, esclarecer ao Cirurgião-dentista os principais fatores relacionados ao surgimento desta complicação. Estudo de Caso: Relata-se o caso de um paciente, gênero feminino, 40 anos, que procurou atendimento afirmando "quando estou comendo, a comida sai pelo meu nariz". Durante a anamnese a paciente informou que há 3 anos foi submetida a exodontia do elemento 26 e que desde então percebeu a passagem de líquidos e alimentos da cavidade oral para a cavidade nasal. Ao exame clínico foi observado um defeito do rebordo alveolar como possível tentativa de reparo fisiológico. Levantou-se a hipótese diagnóstica de fístula buco-sinusal após exodontia do elemento 26 , foi solicitado uma radiografia panorâmica que evidenciou uma descontinuidade da radiopacidade do assoalho do seio maxilar. A resolutividade do caso se deu a partir da prescrição de profilaxia antibiótica e tratamento cirúrgico para fechamento da fístula buco-sinusal pela técnica de retalho vestibular. Paciente encontra-se em acompanhamento odontológico há 3 meses e até o presente momento não apresentou recidivas. Considerações finais: As fístulas buco-sinusais são complicações que podem e devem ser evitadas pelo Cirurgião-dentista através de um planejamento e avaliação detalhada do paciente.

Palavras-Chave: Cirurgia Bucal, Fístula Bucal, Seio Maxilar.

\section{ABSTRACT}

Objective: To clarify the surgical approach of buccal-sinus fistula closure by the buccal flap technique, as well as to clarify to the dentist the main factors related to the onset of this complication. Case Study: We report the case of a female patient, 40 years old, who sought care stating "when I am eating, food comes out of my nose". During the anamnesis, the patient reported that 3 years ago she underwent extraction of the element 26 and since then she noticed the passage of fluids and food from the oral cavity to the nasal cavity. Clinical examination revealed an alveolar ridge defect as a possible attempt for physiological repair. The diagnostic hypothesis of bucco-sinus fistula was raised after extraction of the element 26 , and a panoramic radiograph showing a discontinuity of maxillary sinus floor radiopacity was requested. The resolution of the case was based on the prescription of antibiotic prophylaxis and surgical treatment for buccal-sinus fistula closure by the vestibular flap technique. Patient has been undergoing dental follow-up for 3 months and so far has not relapsed. Final considerations: Oral and sinus fistulas are complications that can and should be avoided by the dentist through detailed patient planning and evaluation.

Key words: Surgery Oral, Oral Fistula, Maxillary Sinus.

\section{RESUMEN}

Objetivo: Aclarar el enfoque quirúrgico del cierre de la fístula del seno bucal mediante la técnica del colgajo bucal, así como aclarar al dentista los principales factores relacionados con la aparición de esta complicación. Estudio de caso: Reportamos el caso de una paciente de 40 años que buscó atención y declaró "cuando estoy comiendo, la comida sale de mi nariz". Durante la anamnesis, la paciente informó que hace 3 años se

${ }^{1}$ Centro Universitário Cesmac, Maceió - AL. *E-mail: flayane23nuberly@outlook.com 
le extrajo el elemento 26 y desde entonces notó el paso de líquidos y alimentos desde la cavidad oral a la cavidad nasal. El examen clínico reveló un defecto de la cresta alveolar como un posible intento de reparación fisiológica. La hipótesis diagnóstica de la fístula buco-sinusal se planteó después de la extracción del elemento 26, y se solicitó una radiografía panorámica que muestra la discontinuidad de la radiopacidad del piso del seno maxilar. La resolución del caso se basó en la prescripción de profilaxis antibiótica y tratamiento quirúrgico para el cierre de la fístula del seno bucal mediante la técnica del colgajo vestibular. El paciente ha estado en seguimiento dental durante 3 meses y hasta ahora no ha recaído. Consideraciones finales: Las fístulas orales y sinusales son complicaciones que el dentista puede y debe evitar mediante una planificación y evaluación detalladas del paciente.

Palabras clave: Cirugía Bucal, Fístula Oral, Seno Maxilar.

\section{INTRODUÇÃO}

O seio maxilar é uma cavidade pneumática localizada no interior do osso maxilar que apresenta dimensão e formato variável. $O$ assoalho do seio maxilar estende-se pelo rebordo alveolar e apresenta íntima relação com os ápices radiculares dos molares, pré molares e eventualmente dos caninos, esta proximidade é um fator determinante no aumento do risco de comunicações da cavidade oral com o seio maxilar durante procedimentos cirúrgicos e consequentemente causando diversas intercorrências (ROQUE-TORRES G, et al., 2016).

As comunicações buco-sinusais ou oro-antrais estão frequentemente relacionadas a exodontia de dentes superiores posteriores, visto que, existe uma íntima relação das raízes dos molares com o seio maxilar. Contudo, outras condições que podem ser descritas como fator etiológico no desenvolvimento desta alteração, como por exemplo o trauma originado pelo uso de instrumentais de forma inadvertida, destruição do seio por deteriorações periapicais e remoção de cistos e até mesmo tumores do palato ou do seio maxilar (BORGONOVO AE, et al., 2012).

Neste contexto, múltiplos procedimento para o fechamento das fístulas buco-sinusais vem sendo relatados na literatura, podendo-se ter como exemplo o deslizamento do retalho vestibular, palatino e/ou combinado. Vale ressaltar que além das técnicas citadas, existem outros métodos possíveis tais como, o uso de inoculações ósseas, implantes utilizando matérias sintéticos e do musculo temporal combinado ao uso do corpo adiposo (GARCIA RR, et al., 2000; RAPIDIS AD, et al., 2000).

A terapêutica para o fechamento da Fístula Buco-sinusal necessita ser realizado o mais preliminarmente possível, para que desse modo seja evitada a infecção do seio e disposição de uma sinusite maxilar. A cirurgia para o fechamento das fistulas buco-sinusais utilizando retalhos vestibulares abrange na atualidade o método mais utilizado para fechamento das comunicações oro-antrais. Esta também é escolhida dentre as demais por sua facilidade na realização da técnica. Caso ocorra a instalação precedente de uma sinusite, é necessário que se proceda a terapêutica da mesma antes do processo cirúrgico (SALIM MMA, et al., 2008).

Portanto, essa comunicação pode ser evitada com o planejamento cirúrgico adequado, exame clínico detalhado e exames de imagem. Em relação ao aspecto cirúrgico, esse fechamento pode ser realizado por técnicas de retalho palatino ou sutura oclusiva, todavia a técnica que será enfatizada no presente trabalho é a técnica de retalho vestibular com ausência de rotatividade do tecido adiposo da bochecha (BORGONOVO $\mathrm{AE}$, et al., 2012).

O objetivo deste estudo foi relatar o caso e elucidar acerca da abordagem cirúrgica do fechamento de fístula buco-sinusal pela técnica de retalho vestibular, bem como, esclarecer ao Cirurgião-dentista os principais fatores relacionados ao surgimento desta complicação.

\section{RELATO DE CASO}

Relata-se o caso de um paciente, gênero feminino, 40 anos, melanoderma, procurou atendimento odontológico na clínica escola do Centro Universitário afirmando "quando estou comendo, a comida sai pelo meu nariz". Durante a anamnese a paciente alegou ser hipertensa, portadora do vírus HIV e usuária de crack 
e que há 3 anos foi submetida a exodontia do elemento 26 e que desde então percebeu a passagem de líquidos e alimentos da cavidade oral para a cavidade nasal.

Durante o exame físico extra bucal não foi identificado nenhuma alteração significativa, contudo, no exame clínico intrabucal foi observado um orifício localizado no rebordo alveolar superior do lado esquerdo na região correspondente a região do primeiro molar superior, apresentando-se como um defeito do rebordo oriundo de possível tentativa de reparo fisiológico (Figura 1).

Figura 1 - Aspecto clínico inicial da fístula.

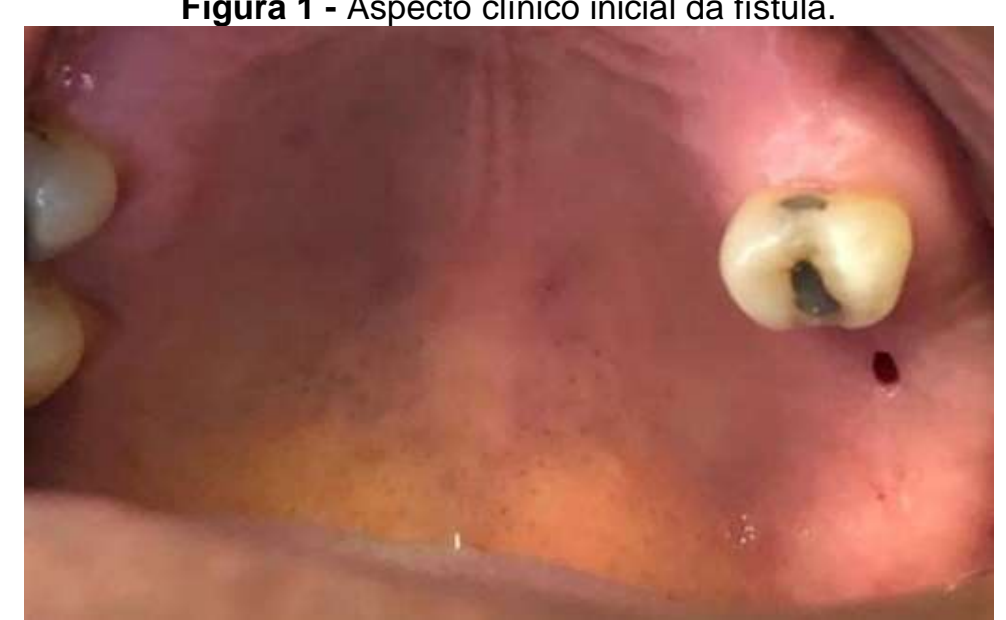

Fonte: Anjos FNFG, Melo MCG, Leite IF, Melo DP, Lucas LA, Cavalcanti TC, Macedo LFC, 2019.

Quando questionada sobre o aparecimento de sintomas característicos da sinusite odontogênica (obstrução, congestão nasal, cefaleia, dor ou pressão na face e coriza), a mesma nega a ocorrência e afirma que o único motivo da busca pelo atendimento odontológico é o desconforto gerado pela saída de líquidos e alimentos pelo nariz.

Com base nos dados coletados através da anamnese e após criterioso exame clínico, levantou-se a hipótese diagnóstica de fístula buco-sinusal após exodontia do elemento 26 , dessa forma, foi realizado a manobra de valsalva (tamponamento nasal acompanhada de estimulação/pressão para saída de ar) onde foi possível observar a saída de ar pelo orifício. Para um maior detalhamento do caso, foi solicitado a realização de uma radiografia panorâmica, que evidenciou uma descontinuidade da radiopacidade do assoalho do seio maxilar, confirmando a hipótese diagnóstica (Figura 2).

Figura 2 - Exame radiográfico panorâmico evidenciando descontinuidade da radiopacidade do seio maxilar.

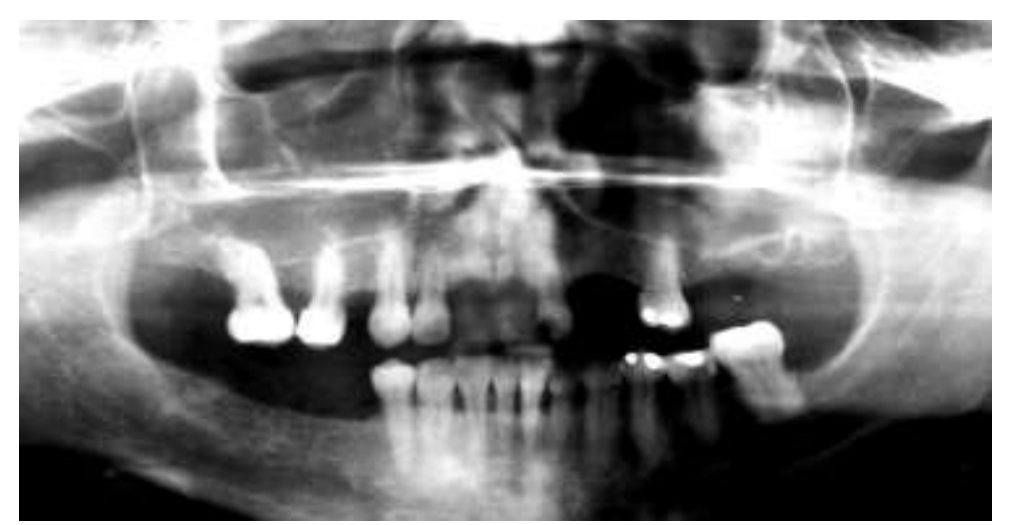

Fonte: Anjos FNFG, Melo MCG, Leite IF, Melo DP, Lucas LA, Cavalcanti TC, Macedo LFC, 2019. 
A conduta clínica inicial foi a explicação quanto a necessidade de suspensão do uso da substância ilícita em um tempo médio de sete dias, prescrição de amoxicilina $500 \mathrm{mg}$ de $8 / 8$ horas por sete dias e utilização de soro fisiológico para irrigação da cavidade nasal com o auxílio de uma seringa de $20 \mathrm{ml}$.

Após sete dias a paciente retornou ao serviço odontológico e para a resolubilidade do caso foi indicada cirurgia para fechamento de fístula buco-sinusal com o uso da técnica do retalho por vestibular. A cirurgia ocorreu sob anestesia local com o bloqueio do nervo alveolar superior posterior e médio com o uso de lidocaína 2\% + epinefrina de 1:100.000 (Alphacaine 2\% 1:100.000, Nova DFL®, Rio de Janeiro, Brasil). Com o auxílio de uma lâmina de bisturi oํ 15 (Swann-Morton, Sheffield Inglaterra) foi realizada uma incisão quadrangular que se estendeu do contorno da fístula até a vertente vestibular da maxila, para obtenção do retalho vestibular, para a diérese do tecido foi utilizado um descolador do tipo molt $n^{\circ} 9$ (S.S. White Duflex®, Rio de Janeiro, Brasil) que possibilitou o descolamento de um retalho muco-periosteal (Figura 3).

Figura 3 - Confecção do retalho vestibular.

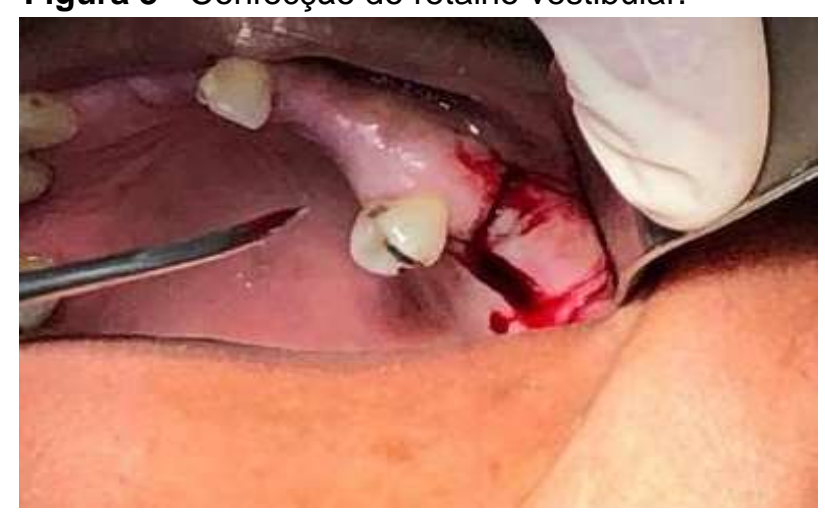

Fonte: Anjos FNFG, Melo MCG, Leite IF, Melo DP, Lucas LA, Cavalcanti TC, Macedo LFC, 2019.

Foi realizado curetagem da mucosa do seio maxilar com o uso de cureta de Lucas (S.S. White Duflex®, Rio de Janeiro, Brasil), seguida de irrigação abundante com soro fisiológico e aspiração.

Para avaliar se a dimensão do retalho era suficiente para o completo fechamento da fístula, foi realizado apreensão do retalho com o uso de uma pinça dente de rato (Golgran ${ }^{\circledR}$, Brasil) e tracionamento para a área a ser recoberta (Figura 4A). Em seguida foi realizada síntese com o uso de Ethicon Mononylon 3.0 (Johnson \& Johnson ${ }^{\circledR}$, São Paulo, SP, Brasil) em múltiplos pontos simples isolados (Figura 4B).

Figura 4 (A, B): Tracionamento e síntese para estabilidade do retalho.

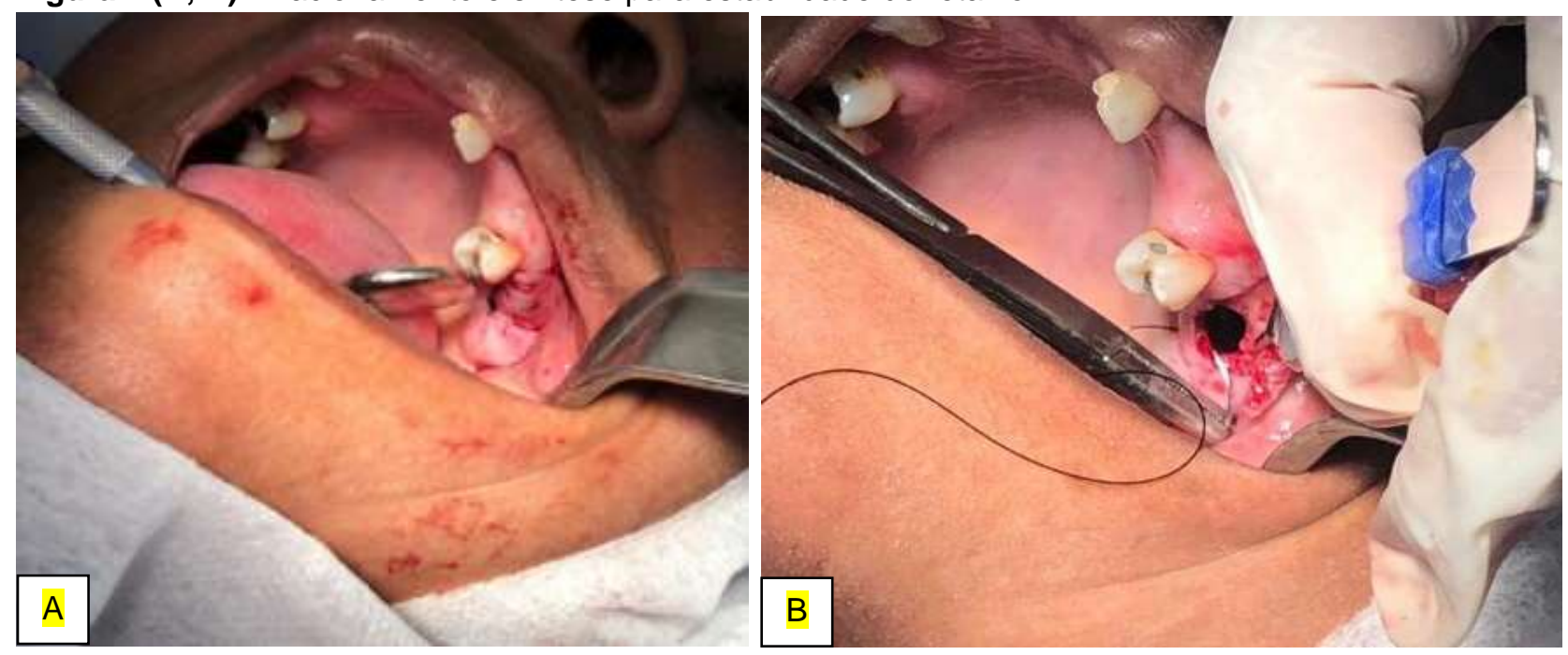

Fonte: Anjos FNFG, Melo MCG, Leite IF, Melo DP, Lucas LA, Cavalcanti TC, Macedo LFC, 2019. 
Para o pós operatório a paciente foi instruída a abster-se do uso do crack, foi prescrito amoxicilina $500 \mathrm{mg}$ e o nimesulida $100 \mathrm{mg}$ por 7 dias, além disso, recebeu informações para os cuidados do pós operatório, sendo estes, evitar assoar o nariz, evitar comidas muito quentes e picantes, não mastigar na região, evitar uso de bochechos, não fumar, não beber e não realizar nenhum exercício ou esforço físico. A remoção da sutura foi realizada com 7 dias do ato operatório.

Em um período de 30 dias, a paciente retornou ao serviço odontológico para reavaliação da condição final alegando ausência de sinais e sintomas. Durante o exame clínico intrabucal é perceptível o fechamento completo da fístula (Figura 5).

Figura 5 - Aspecto final (após 30 dias).

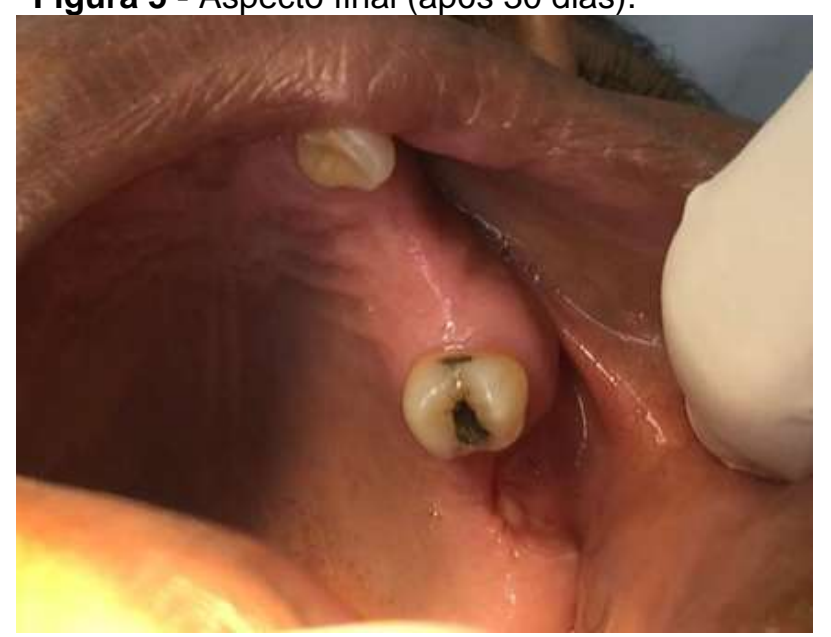

Fonte: Anjos FNFG, Melo MCG, Leite IF, Melo DP, Lucas LA, Cavalcanti TC, Macedo LFC, 2019.

Paciente encontra-se em acompanhamento clínico na clínica escola do Centro Universitário há três meses e até o presente momento não apresenta sinais de recidiva da fístula. A paciente foi encaminhada para acompanhamento pelo Centro de Atenção Psicossocial (CAPS), para abster-se do uso de substâncias ilícitas, pois isto é um fator que influenciará na sua completa recuperação.

\section{DISCUSSÃO}

De acordo com a literatura, a presença de comunicação buco-sinusal muitas vezes se dá pela exodontia de elementos localizados na parte posterior da maxila, no presente relato, a paciente apresenta edentulismo parcial, com várias perdas dentárias na parte posterior da maxila, incluindo o primeiro molar superior, sendo este, fator etiológico da fístula. Ao se comparar a incidência das fístulas buco- sinusais em relação a gêneros, enfatiza-se uma maior predominância pelo gênero masculino, principalmente durante a vida adulta, no caso relatado ocorreu em gênero feminino, tendo assim uma divergência com a literatura. Tratando-se do que pode vir a ocasionar esta fístula, pode-se citar iatrogênia com uso de instrumentos e manobras inadequadas de curetagem durante exodontias de elementos dentários superiores posteriores próximo ao seio maxilar. Esta perfuração é mais propicia em dentes com raízes divergentes, próximos a espaços ausentes de elementos dentários devido a suscetibilidade da fibromucosa, tomando assim o alvéolo fragilizado e expondo a cavidade sinusal (GARCIA RR, et al., 2000).

A literatura afirma que uma vez realizada a comunicação buco-sinusal, é de suma importância seu fechamento precoce, caso contrário tem-se a instalação da sinusite maxilar como complicação patológica, sendo assim divergindo da literatura, o presente relato expõe que após três anos com a comunicação, não houve sinais de sinusite. $O$ procedimento cirúrgico da fístula buco-sinusal deverá esperar em casos de infecções da região, para que sejam totalmente eliminadas. O local com maior predominância para o seu surgimento é um assunto amplamente discutido entre os autores. Não existe um acordo sobre as indicações 
das técnicas para o tratamento desta complicação patológica. Assim, possuindo o resultado, deve-se analisar a extensão da comunicação, visto que o tratamento está diretamente relacionado ao tamanho da abertura (SCATARELLA A, et al., 2010).

A indicação destas técnicas irá depender de cada caso, a técnica do retalho palatino rodado tem como objetivo o fechamento da fístula devido ao procedimento mal sucedido da técnica vestibular. Tendo como finalidade a reparação tardia da mesma, possuindo dentre suas vantagens uma boa vascularização, fácil utilização, espessura adequada, apresentando um tecido espesso. Dentre suas desvantagens destaca-se, a viabilidade do tecido circundante vir a sofrer processo de necrose (SILVEIRA RL, et al., 2008).

Em relação a técnica do corpo adiposo, vale ressaltar que só poderá ser realizada em pacientes que possuam o corpo adiposo, tendo-se uma parcela da população, que por questões estéticas optam por sua remoção, a mesma localiza-se próximo ao musculo bucinador. Devido a sua localização anatômica tem-se assim um acesso facilitado na confecção do retalho e possibilita seu uso em diferentes tamanhos e localizações variada, possuindo um bom prognostico e boa vascularização já que não interfere no fundo do sulco vestibular, evitando assim, possíveis recidivas, apresenta como vantagens ser de fácil utilização e escolhida quando trata-se de grandes feridas cirúrgicas e suas desvantagens trata-se de trismo no pósoperatório e podendo apresentar alterações estéticas, causadas por defeitos na musculatura e fonética apresentando problemas na fala (PARISE GK e TASSARA LFR, 2016).

A utilização com enxertos ósseos é inovadora devido a não utilização de tecidos moles, tendo a possibilidade de ser utilizado em ofícios médios e extensos. Tendo sua principal vantagem evitar possíveis efeitos colaterais que poderão ocorrer com a utilização de outras técnicas. Por se tratar de uma técnica nova e não ter repercussão, tem-se isto como uma desvantagem (SCATARELLA A, et al., 2010).

A utilização de retalhos vestibulares engloba na atualidade a técnica cirúrgica mais utilizada para o fechamento de fístulas buco-sinusais, devido à facilidade de realização, pouca morbidade, e sua possibilidade de utilização sob anestesia local. Este retalho também deixa uma área menos cruenta, possuindo boa vascularização em comparação aos retalhos palatinos. Os retalhos vestibulares propiciam uma redução do fundo do vestíbulo que subsequente irá necessitar de uma nova intervenção cirúrgica para sua remodelação (SALIM MAA, et al., 2008).

Aliado a exames complementares e uma boa técnica cirúrgica, além dos cuidados pré-operatórios, uma boa profilaxia medicamentosa e considerando a individualidade de cada paciente, estas elevam as chances de se evitar complicações pós-cirúrgicas, como infecções. Para se chegar a um diagnóstico eficaz e conclusivo da fistula buco-sinusal são necessários exames de imagens, e aliado a estes a manobra de valsalva, esta constitui na realização do fechamento nasal e abertura bucal, tampando o ouvido médio. Sendo a radiografia panorâmica uma excelente escolha para o correto diagnóstico, devido a esta ter imagens precisas e gerais de toda extensão da face e uma maior visão da descontinuidade do seio maxilar (SILVEIRA $\mathrm{RL}$, et al., 2008).

\section{CONSIDERAÇÕES FINAIS}

Cabe ao cirurgião dentista realizar uma anamnese de forma detalhada e precisa. O profissional deve solucionar esta comunicação o quanto antes, pois deixando-a abertura por muito tempo pode-se levar à infecção do seio, instalando-se uma sinusite maxilar, ocorrendo um agravamento do caso e assim instalando outras patologias.

\section{REFERÊNCIAS}

1. BORGONOVO AE, et al. Surgical Options In Oroantral Fistula Treatment, Italy. The Open Dentistry Journal, 2012; v. 6.

2. COSTA MDMA. Extração de Terceiros Molares: Perfil do atendimento em clínicas de cirurgia da graduação e especialização da faculdade de odontologia da universidade federal de Uberlândia. Dissertação (Mestrado em odontologia) - Faculdade de Odontologia da Universidade Federal de Uberlândia, Uberlândia, 2008; 80 p. 
3. GARCIA RR, et al. Utilização de enxerto pediculado do corpo adiposo da bochecha no tratamento de comunicações oro-antrais. Rev Port Estomatol Cir Maxilofac 2000; 41: 17-24.

4. HANAZAWA Y, et al. Closure of oroantral communications using a pedicled buccal fat pad graft. São Paulo. J Oral Maxillofac Surg 1995; 53: 771-5.

5. KRAUSE CF, et al. Manejo quirúrgico de la fístula oroantral. Chile. Rev otorrinolaringo Cir cabeza cuello 1999; 59(2):101-7

6. MARTIN-GRANIZO R, et al. Use of buccal fat pad to repair intraoral defects: review of 30 cases. Br J Oral Maxillofac Surg, 1997; 35: 81-4.

7. PARISE GK, TASSARA LFR. Tratamento Cirúrgico e Medicamentoso das Comunicações Buco-Sinusais: Uma Revisão de Literatura. Madrid. Universidade Regional Integrada do Alto Uruguai e das Missões - URI Erechim, 2016; p. 10.

8. RAPIDIS AD, et al. The use of the buccal fat pad for reconstruction of oral defects: review of the literature and report of 15 cases. Grécia. J Oral Maxillofac Surg 2000; 58: 158-63.

9. SALIM MAA, et al. Tratamento de fístula buco-sinusal: revisão de literatura e relato de caso clínico. Rev. Bras. Odontol., Rio de Janeiro, v. 65, n. 1, p. 101-105, jan./jun. 2008.

10. SCATARELLA A, et al. Treatment of oroantral fistula with autologous boné graft and application of a nonreabsorbable membrane. Italy. Int. J. Med. Sci., v. 7, n. 5, p. 267-271. 2010.

11. SCHOW SR. Doenças odontogênicas do seio maxilar. In: Peterson LJ et al. Cirurgia oral e maxilofacial contemporânea. $2^{a}$ ed. Rio Janeiro: Guanabara Koogan; 1997. p.465-77.

12. SILVEIRA RL, et al. Tratamento de fístula bucosinusal através de retalho palatino. Rev. Cir. Traumatol. Buco-Maxilofac., Camaragibe, v. 8, n. 1, p. 29-34, jan./mar. 2008.

13. ROQUE-TORRES GR, et al. Relação entre doenças no seio maxilar e dentes hígidos. Piracicaba. Brazilian Journal of Otorhinolaryngology, 2016; 82(1), 33-38.

14. MENESES RO, et al. Deslocamento de resto radicular no seio maxilar devido a manobras incorretas de exodontia: um relato de caso. Camaragibe, 2014; Ver. Cir. Traumatol. Buco-Maxilo-fac.,14, p.4.

15. FARIAS JG, CÂNCIO AV, BARROS LF. Fechamento de fístula bucossinusal utilizando o corpo adiposo bucal Técnica convencional x técnica do túnel - Relato de casos clínicos. Camaragibe Rev. Cir. Traumatol. Buco-Maxilofac., $2015 ; 15$, n.3.

16. RALDI FV, et al. Fechamento de Comunicações Buco-Sinusais. Porto Alegre. RGO, 2005; 54: 178-181. 